\title{
Minimum Spanning Tree based Improved Routing Protocol for Heterogeneous Wireless Sensor Network
}

\author{
Risha Vashist \\ Research scholar \\ Chandigarh Engineering College, \\ Landran
}

\author{
Suniti Dutt \\ Assistant Professor \\ Chandigarh Engineering College, \\ Landran
}

\begin{abstract}
Performing multiple tasks like data aggregation, processing and communication to other Sensor Nodes (SNs) or Base Station (BS) is a very attractive and effective attribute carried out by Wireless Sensor Networks (WSNs). As SNs have limited energy resources, storage capabilities and processing, the network layer protocol has therefore to guarantee reliable communication under these circumstances. Literature survey implies that hierarchical clustering and node heterogeneity are two key parameters that can ensure the enhanced lifetime of SNs. Keeping this in mind Improved Heterogeneous Routing Protocol (IHRP) is proposed. IHRP evenly distributes the load among clusters to ensure good network lifetime. A comparison is made between the performances of IHRP and Enhanced Heterogeneous Low Energy Adaptive Clustering Hierarchy (EHE-LEACH). Simulation results throw light on the improvement in lifetime of EHE-LEACH protocol by introducing this load balancing algorithm IHRP.
\end{abstract}

\section{General Terms}

Networks, Wireless

\section{Keywords}

Sensor Networks, network lifetime, heterogeneous, clustering, minimum spanning tree.

\section{INTRODUCTION}

Random deployment of SNs in large number forms a Wireless Sensor Network (WSN). WSNs witnessed a tremendous development in different areas with passage of time. Distribution can vary from a hundred to thousand nodes spread over a wide area network. A WSN basically consists of a number of nodes better referred to as a sensor node. These nodes are usually built of parts like radio transceiver with an antenna, microcontroller, power supply and the actual sensor. This sensor is actually used to sense, measure and gather information from the environment to transfer the sensed data to the user [1]. Applications of a WSN include fields like forest fire detection and air pollution monitoring. Landslide detection and water quality monitoring also come under the applications of a WSN.

Minimizing the energy consumption is one of the major tasks or requirement in a WSN. In order to improve the lifetime of a network it is mandatory that it has a good energy efficiency [1,2]. Improving the lifetime of a network will yield a better network by increasing the lifetime of sensor nodes. Routing basically reflects the way in which the sensing nodes direct their data to communicate with the sink. Hierarchical routing protocols are based on clubbing the sensor nodes into clusters thereby forming many such small clusters to address the weaknesses of flat routing protocols like low network lifetime and low efficiency. Energy loss increases as the sensor node directly sends the sensed data to the remote base station. Sensor nodes located at a large distance from the BS tend to lose energy prior to ones situated near the base station [3]. In case of Minimum Transmission Energy (MTE), sensor nodes near the BS use energy much before the rest as they participate solely in routing the data messages sent by the other nodes to the BS $[2,3]$.

With time many other routing protocols have been suggested such as Data-Centric routing for WSN which includes protocols like (SPIN) [4], Directed Diffusion (DD) [5,6]. In spite of providing robustness and reliability, data-centric protocols inhibit some shortcomings. SPIN and DD sustain loss of energy resources because of the overhead caused during requesting, advertising and setting up the gradient.

In order to overcome the shortcomings stated above the following approach has been laid by handling the load in a Heterogeneous environment. Homogeneous WSNs are different as compared to Heterogeneous WSNs (HWSNs) in terms of sensing, communication, energy and computation. HWSNs generally encounter four types of heterogeneity summarized below [7]:

$>$ Energy Heterogeneity, which refers to different energy levels of various nodes.

$>$ Computational Heterogeneity, that points at different data storage capacities and data compression techniques.

$>$ Communication Heterogeneity includes varied transmission rates and communication ranges.

$>$ Sensing Heterogeneity, that points at different sensing ranges and variation in size of data packets.

Here Improved Heterogeneous Routing Protocol (IHRP) has been proposed to extend the network lifetime. In this approach clusters are formed followed by finding routing paths including Cluster Head $(\mathrm{CH})$ rotation. IHRP focuses on maintaining equal load in all clusters to prevent the overloading of $\mathrm{CHs}$ and incorporates use of Multi-hop for head-to-head routing of sensed information to remote BS.

Organization of paper is carried out as follows: Section II contains the related work done Section III highlights the proposed protocol. Section IV includes the simulation results followed by conclusions, future work and references.

\section{RELATED WORK}

\section{A. $L E A C H$}

LEACH (Low Energy Adaptive Cluster Hierarchy) [3] was first introduced by W.R. Heinzelman. It is one of the most popular hierarchical algorithms. In this, the $\mathrm{CH}$ election and the cluster formation is done randomly. To evenly distribute the energy dissipation in the cluster, the role of $\mathrm{CH}$ is hence rotated. The major drawback of this protocol lies in the fact 
that the residual energy of sensor nodes is not considered and zero energy consumption is considered for formation of cluster.

\section{B. PEGASIS}

An enhancement over LEACH is PEGASIS (Power Efficient Gathering In Sensor Information Systems [8]. It provides 100$300 \%$ lifetime intensification aloft LEACH. Here the $\mathrm{CH}$ election is random from the dedicated chain and is in charge of data transmission to the base station. Drawback associated with this algorithm is that it requires the global knowledge of the network. Also in PEGASIS there is a significant delay since the data has to be communicated in the chain and $\mathrm{CH}$ waits until all the messages are received before transmitting further to the BS.

\section{SEP}

Smaragdakis et al. [9] introduced Stable Election Protocol (SEP) for a two level heterogeneous network which incorporates two types of nodes. In SEP election probabilities are weighted by initial energy of the node relative to that of other nodes in the network. SEP is scalable as it does not crave for any knowledge of exact location of each node. Disadvantage of SEP is that it does not engage well in terms of endurance or stability for multi-level heterogeneous networks.

\section{DEEC and SDEEC}

Distributed Energy-Efficient Clustering (DEEC) [10] is another protocol based on LEACH. Two levels of heterogeneity are taken in this algorithm and then a general solution for multi-stage heterogeneity is approached for. Stochastic DEEC (SDEEC) is an improvement of this protocol. Like DEEC this protocol takes into account twolevel heterogeneity but it conserves energy by making non $\mathrm{CH}$ nodes sleep, unlike DEEC. It also divides the sensor network into dynamic clusters.

\section{E. EHE-LEACH}

EHE-LEACH (Enhanced Heterogeneous (LEACH) [11] protocol for lifetime enhancement of WSNs is based on fixed distance threshold used for the bifurcation of direct communication and cluster based communication. In this research focus is laid on the constraints of energy of individual sensor node and complete network both. Observations show that EHE-LEACH has a better system lifetime and stability as compared with LEACH and SEP.

\section{F. $B C D C P$}

Based Station Controlled Dynamic Clustering Protocol (BCDCP) [12] is another clustering based routing protocol which makes use of BS having a large energy to create clusters. BCDCP improves LEACH by two aspects. Firstly it uses Minimum Spanning Tree (MST) to connect $\mathrm{CH}$ and randomly chooses a leader to send data to BS. Also it makes best use of high energy BS to choose $\mathrm{CHs}$ and make clusters by iterative cluster splitting algorithm. Thus it reduces more energy dissipation than LEACH. Although in small scale network it works well to route data efficiently but it is not appropriate for long distance communications.

\section{PROPOSED PROTOCOL}

This paper proposes an approach called Improved Heterogeneous Routing Protocol (IHRP) algorithm whose main aim is to increase the network lifetime of the Heterogeneous WSN. Vital operations are carried out by BS to form clusters having approximately balanced count of sensor nodes to maintain load in every cluster.

\subsection{Network Model}

Considered radio dissipation model is shown in the figure below. Radio electronics is run by the transmitter which dissipates energy and the receiver dissipates energy to run the radio electronics. If the communication distance is however less than distance $d_{o}$ free space channel model is used otherwise multipath model is followed.

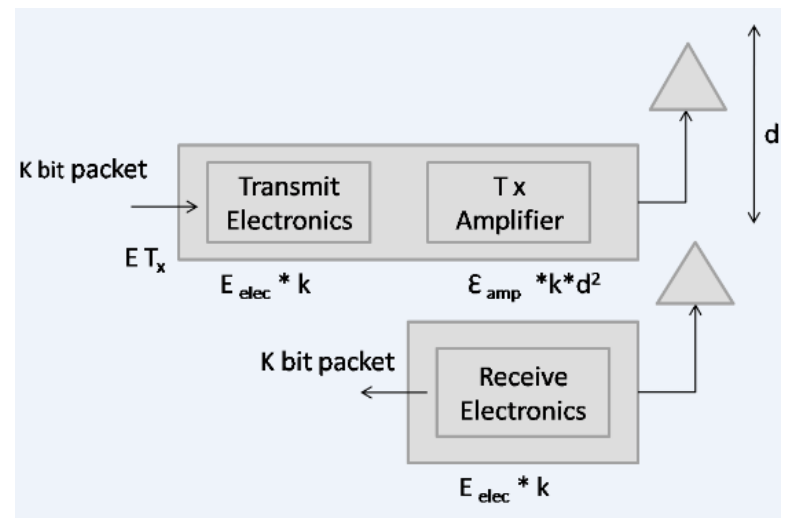

Fig 1. Radio energy dissipation model

Therefore in order to transmit a k-bit message over a distance $d$ using this radio model, transmission energy is:

$E_{T x}(k, d)=k * E_{\text {elec }}+k * \varepsilon_{a m p} * d^{n}$

Where pathloss component $n$ (2 or 4) and also the amplification factor $\varepsilon_{a m p}\left(\varepsilon_{f s}\right.$ or $\left.\varepsilon_{m p}\right)$ are defined in the following equation set for free and multipath environment respectively.

$E_{T x}(k, d)=\left\{\begin{array}{c}k * E_{\text {elec }}+k * \varepsilon_{f s} * d^{2},\left(d<d_{o}\right) \\ k * E_{\text {elec }}+k * \varepsilon_{m p} * d^{4},\left(d \geq d_{o}\right)\end{array}\right\} \ldots$

$E_{\text {elec }}$ is the transmitter circuitry dissipation per bit

$d_{o}=\sqrt{\frac{\varepsilon_{f s}}{\varepsilon_{m p}}}$, Receiving cost,

$E_{R x}(k)=E_{\text {elec }} * k \ldots \ldots$ (3)

\subsection{Two-level Heterogeneity}

There are two types of nodes associated with two-level heterogeneity. These are the normal nodes and advanced nodes. Advanced nodes have more initial energy in comparison to normal nodes. Assume ' $\mathrm{m}$ ' is the fraction of total number of nodes $\mathrm{N}$ having ' $\mathrm{a}$ ' times more energy than normal nodes. These nodes represent the advanced nodes and remaining $\mathrm{N}^{*}(1-\mathrm{m})$ are the normal nodes. The total energy of the network varies as there are two different types of nodes deployed. If $E_{O}$ is the initial energy of each normal node then the energy of each advanced node is $E_{O} *(1+$ a). Heterogeneity model used is same as taken in SEP. Therefore total initial energy of the network rounds off to $\mathrm{N} * E_{O} *(1+\mathrm{a} * \mathrm{~m})$

\subsection{Minimum Spanning Tree [MST]}

Assume an undirected, connected graph. A spanning tree of that graph would be a sub graph that connects all the vertices of that tree. A single graph can have many spanning trees. If some weights or numbers are assigned to each edge, then the MST is the spanning tree with weight less than any other 
spanning tree of that graph. A graph is shown in Figure 2. Weights are assigned to each of its branches. As is seen the highlighted tree is known as the spanning tree. Many other spanning trees can also be built by connecting the vertices of the given figure in different ways.

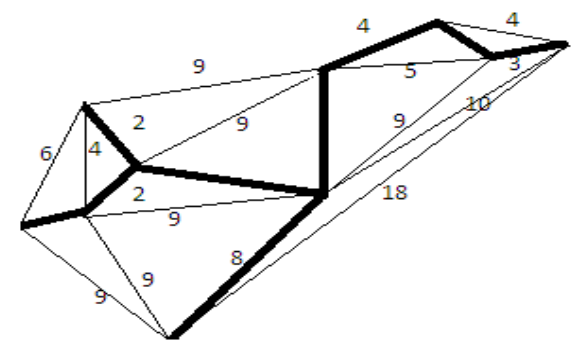

Fig 2. Edge weighted graph and its MST

\subsection{Working}

Improved Heterogeneous Routing Protocol (IHRP) is carried out in three phases:

\section{Initial Phase \\ 2. Setup Phase \\ 3. Data Transmission Phase}

INITIAL PHASE: The sensor nodes are deployed in the sensing area, where each node is heterogeneous in terms of energy. During this phase the BS sets details of geographical positioning and residual energy of all the nodes. Hence, allowing the BS to compute the relative distance between the nodes and to the BS.

SETUP PHASE: The core activities in this phase are cluster formation, $\mathrm{CH}$ election, forming MST routing path for $\mathrm{CH}$-to$\mathrm{CH}$ and $\mathrm{CH}$-to-forwarding $\mathrm{CH}$ and creating schedule for each cluster. With start of each setup phase, nodes send the current energy status to the BS. The Cluster formation algorithm forms a group of clusters by considering distances between all the sensor nodes. Load Balancing is executed at BS. It refers to maintaining a balanced number of sensor nodes in each cluster. A Distance Matrix between all the $\mathrm{N}$ corresponding sensor nodes is computed (using the Euclidean distance). The two most distant nodes are obtained from the step1 known as border nodes, BN1 and BN2. ClusterA and ClusterB groups are formed thereafter. One group comprises of $\mathrm{BN} 1$ and $k-1$ sensor nodes which are closest to $\mathrm{BN} 1$. The other group consists of $\mathrm{BN} 2$ and $k-1$ sensor nodes nearest to $\mathrm{BN} 2 . k$ is the optimum number of nodes a cluster has in order to maintain balance between clusters, $k$ value can be obtained since for optimal network maximum 5\% of all sensor nodes can become $\mathrm{CH}$. Rest of the sensor nodes which do not belong to any of the two groups formed before, repeat the procedure and compute $k$ again to balance the clusters. If there are less than $k$ sensor nodes which do not belong to any of the formed cluster, they form a new group of cluster. Upon completion of Load Balancing algorithm each cluster contains approximately equal number of nodes, their by load per cluster is balanced throughout the network. The second core activity in the setup phase is construction of MST route in between all $\mathrm{CHs}$ and $\mathrm{CH}$-to-forwarding $\mathrm{CH}$. The proposed protocol adopts Multi-hop scheme in order to transfer the sensed data to the distant BS.

DATA TRANSMISSION PHASE: After the setup phase, the next is the data transmission phase. Each sensor node transmits the sensed data to their respective $\mathrm{CHs}$ based on the TDMA (Time Division Multiple Access) schedule created in the previous phase. After receiving all the data from the sensor nodes the $\mathrm{CH}$ perform data fusion including the other necessary information required by the BS. It is finally forwarded to the forwarding $\mathrm{CH}$ through $\mathrm{CH}$-to- $\mathrm{CH}$ routing paths and then fused data is transmitted to the BS.

\section{SIMULATIONS AND RESULTS}

This work assumes that 100 sensor nodes are randomly scattered in a two-dimensional square field of dimensions $100 \times 100$ square meters. For the purpose of analysis, MATLAB is used to implement the simulation. The network parameters are summarized in Tables 1 and 2.

TABLE 1: Parameter Settings

\begin{tabular}{|l|l|}
\hline \multicolumn{1}{|c|}{ Parameters } & \multicolumn{1}{|c|}{ Value } \\
\hline Network size (square meters) & $100 \mathrm{X} 100$ \\
\hline Location of base station (meters) & 100,100 \\
\hline Number of nodes & 100 \\
\hline Data packet length (bits) & 4000 \\
\hline Threshold distance (meters) & 87 \\
\hline $\begin{array}{l}\text { Transmitter/Receiver Electronics } \\
\text { Energy }\end{array}$ & $50 \mathrm{nj} / \mathrm{bit}$ \\
\hline Data Aggregation Energy & $5 \mathrm{nj} / \mathrm{bit}$ \\
\hline $\begin{array}{l}\text { Transmitter Amplifier Energy, } \varepsilon_{f s}, \\
\text { if } d_{t o} B s \leq d_{o}\end{array}$ & $10 \mathrm{pJ} / \mathrm{bit} / \mathrm{m} 2$ \\
\hline $\begin{array}{l}\text { Transmit Amplifier Energy, } \varepsilon_{a m p}, \\
\text { if } d_{t o} B S \geq d_{o}\end{array}$ & $0.0013 \mathrm{pJ} / \mathrm{bit} / \mathrm{m} 4$ \\
\hline Optimal Probability & 0.5 \\
\hline
\end{tabular}

TABLE 2: Two level Heterogeneity

\begin{tabular}{|l|r|}
\hline \multicolumn{1}{|c|}{ Parameters } & Value \\
\hline Proportion of advanced nodes, $\mathrm{m}$ & 0.1 \\
\hline $\begin{array}{l}\text { Energy factor for advanced nodes, } \\
\text { a }\end{array}$ & 1 \\
\hline $\begin{array}{l}\text { Initial energy of normal nodes, } \\
\text { joules }\end{array}$ & 2 \\
\hline
\end{tabular}

\subsection{Analysis of Experiment}

Network Lifetime: Network Lifetime is considered in terms of Half Nodes Alive (HNA) and Last Node Alive (LNA). Half Nodes alive mean that $50 \%$ of the nodes are dead and LNA means a single node is alive in the network. Performance is measured in terms of network lifetime. During simulation, different random sensor network topologies have been considered which contain 100 nodes 


\subsection{Comparing proposed protocol IHRP with EHE-LEACH}

The deployment of heterogeneous sensor nodes in the WSN is shown in Figure 3, here a normal node is denoted by a 'o' and an advanced node is denoted by a ' + '. The BS is at the corner of the field depicted by ' $x$ '. In this case there are 10 advanced nodes deployed with 2 times more energy than normal nodes. The rest 90 nodes are normal nodes.

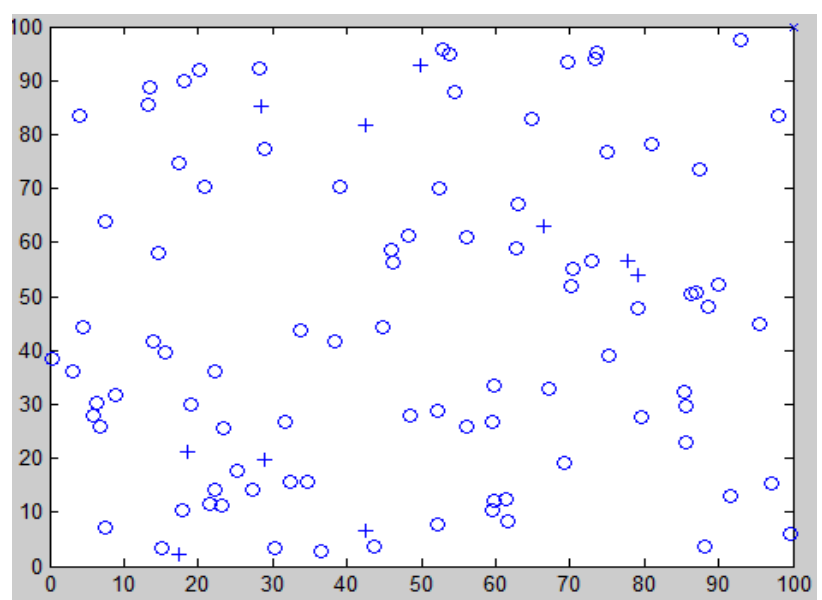

Fig 3. Deployment of Two-level HWSN

Figure 4 represents cluster formation of the 100 nodes taken into account by indicating each cluster with a different color. It also highlights the formation of a MST that represents the minimum spanning distance or route taken to transmit the sensed data to the BS. In order to obtain the performance of IHRP, IHRP protocol is simulated using Matlab and compared with another cluster-based routing protocol EHELEACH.

In the Figures 4(i) and 4(ii) it is clearly evident that there are different cluster formations for different number of iterations because of random sensor network creation at each iteration. Red line indicates the joining of $\mathrm{CHs}$ which compute the route to transfer the data to the BS.

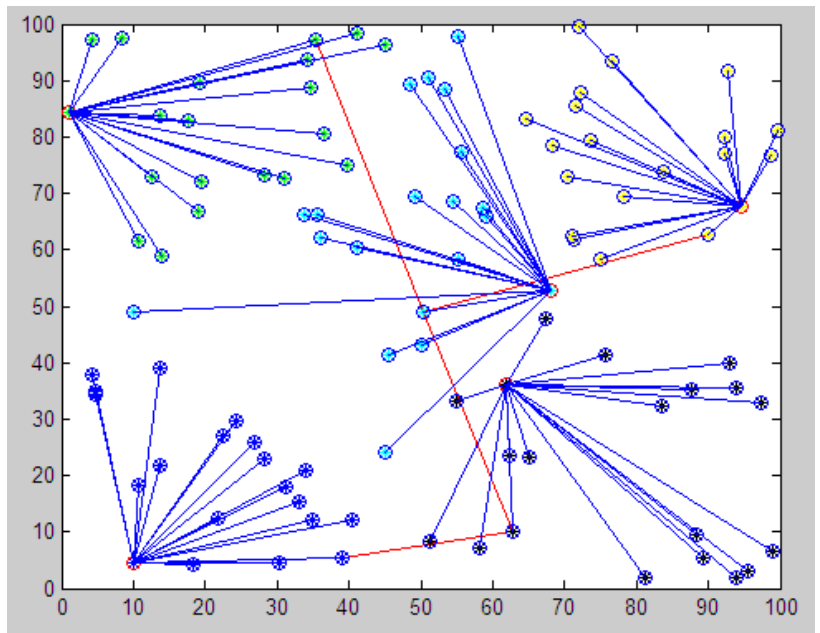

Fig 4(i). Building MST

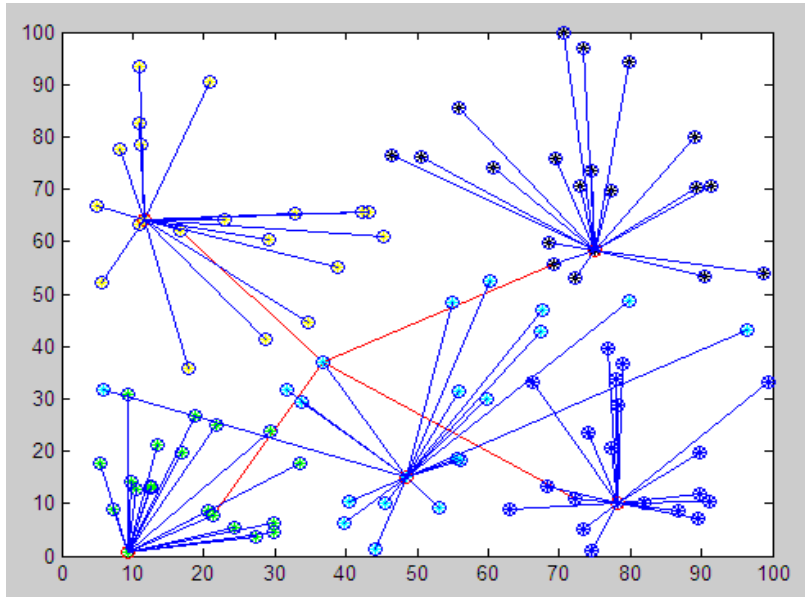

Fig 4(ii). Building MST

Figure 5 and Figure 6 show the lifetime analysis of EHELEACH and IHRP protocols and hence compare them on the basis of HNA and LNA respectively.

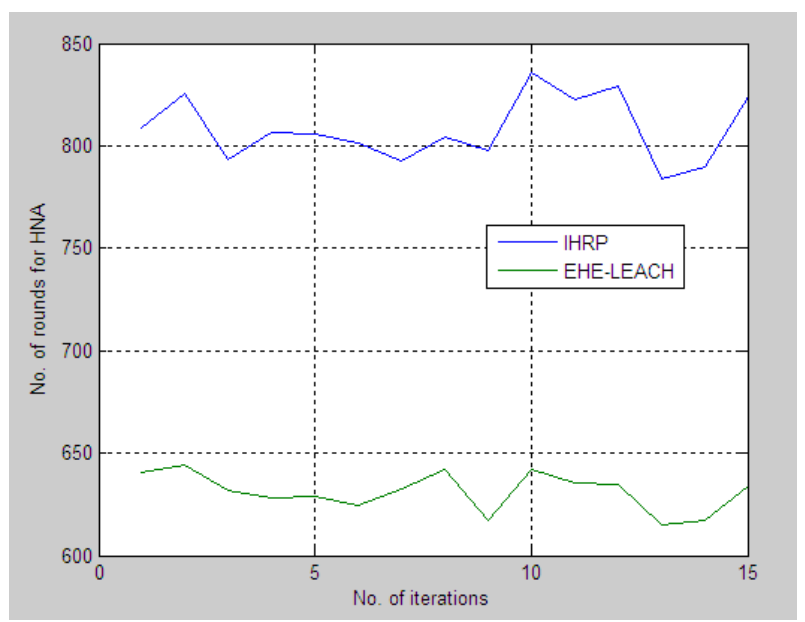

Fig 5. Network lifetime in terms of HNA

Figure 5 represents the comparison of lifetime between IHRP and EHE-LEACH on the basis of HNA. It basically defines the number of rounds that are taken when half nodes of the network perish away for fifteen different iterations. As is seen from the figure, it depicts that on the $5^{\text {th }}$ iteration half nodes remain alive on the $625^{\text {th }}$ round in case of EHE-LEACH. Similarly, for $10^{\text {th }}$ and $15^{\text {th }}$ iterations number of rounds reached by half of the nodes is 640 and 630 . In case of IHRP half nodes die at round 810 for $5^{\text {th }}$ iteration following 835 and 825 for $10^{\text {th }}$ and $15^{\text {th }}$ iterations. This depicts an improvement of about $30.3 \%$ over the EHE-LEACH protocol.

Figure 6 also compares the lifetime between the two protocols but in terms of LNA. It represents the number of rounds that are completed before the last node dies. Fifteen iterations are taken into notice to account for random network creation in each iteration. As per the figure EHE-LEACH takes 1410 rounds when the last node is only left. $10^{\text {th }}$ iteration shows the number to be 1450 and $15^{\text {th }}$ iteration completes 1260 rounds. Now looking for IHRP it is seen that it completes 1510, 1480 and 1530 rounds for LNA in $5^{\text {th }}, 10^{\text {th }}$ and $15^{\text {th }}$ iterations respectively. Hence percent improvement for LNA reaches around $24.7 \%$ on an average. 


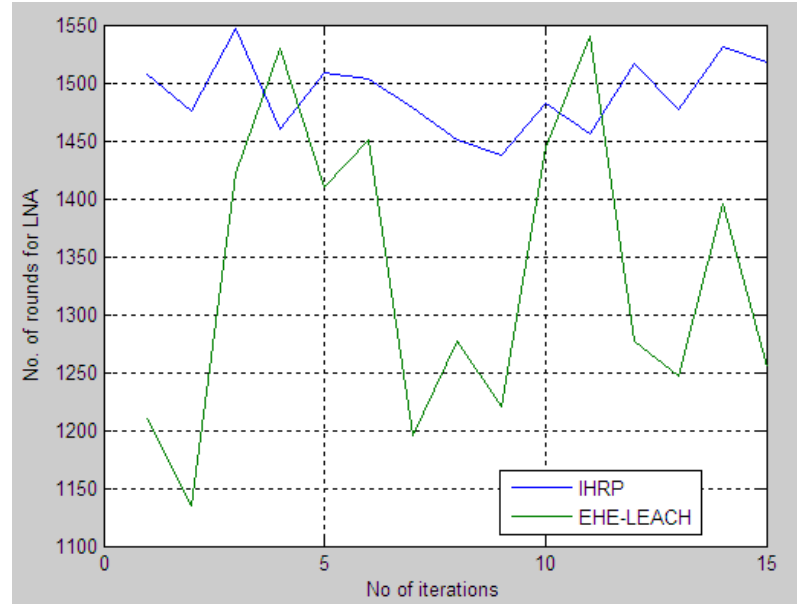

Fig 6. Network lifetime in terms of LNA

\section{CONCLUSION AND FUTURE SCOPE}

Researchers have previously worked towards extending the lifetime of WSNs by adopting many techniques but there is still a need to develop more robust designs for heterogeneous networks. Generally there exists no obvious definition for lifetime. Here HNA and LNA are used as the parameters to express lifetime measurement and stability. The network lifetimes improved by $30.3 \%$ and $24.7 \%$ in terms of HNA and LNA. In this work an Improved Heterogeneous Routing Protocol is proposed for WSNs. The key concept used here was to maintain balanced number of nodes in each cluster by adopting multi-hop scheme using MST approach. Thus by considering simulation results it is concluded that the proposed protocol IHRP stands better in terms of performance as compared to EHE-LEACH protocol.This work can be further developed for three-level heterogeneity. Load balancing and stability can be further studied by considering a large area placing more number of nodes.

\section{REFERENCES}

[1] F. Akyildiz, W. Su, Y. Sankarasubramaniam, and E. Cayirci, "A survey on sensor networks," IEEE Communications Magazine, vol. 40, no. 8,pp. 102-114, 2002 .

[2] W. B. Heinzelman, A. P. Chandrakasan, and H. Balakrishnan, "An Application-Specific Protocol Architecture for Wireless Microsensor Networks," IEEE Trans. Wireless Commun., vol. 1, no. 4, Oct. 2002, pp.660-70.

[3] W. R. Heinzelman, A. P. Chandrakasan, and H. Balakrishnan, "Energy Efficient Communication
Protocol for Wireless Microsensor Networks",Proc. 33rd Hawaii Int'l. Conf. Sys. Sci., Jan. 2000.

[4] W. Heinzelman, J. Kulik, H. Balakrishnan, Adaptive protocols for information dissemination in wireless sensor networks, in: Proceedings of the 5th Annual ACM/IEEE International Conference on Mobile Computing and Networking (MobiCom_99), Seattle, WA, August 1999.

[5] C. Intanagonwiwat, R. Govindan, D. Estrin, Directed diffusion: a scalable and robust communication paradigm for sensor networks, in: Proceedings of the 6th Annual ACM/IEEE International Conference on Mobile Computing and Networking (MobiCom_00), Boston, MA, August 2000.

[6] D. Estrin et al., Next century challenges: scalable coordination in sensor networks, in: Proceedings of the 5th annual ACM/IEEE InternationalConference on Mobile Computing and Networking (MobiCom_99), Seattle, WA, August 1999.

[7] M. Yarvis, N. Kushalnagar, H. Singh, "Exploiting heterogeneity in sensor networks", Proceedings of 24th Annual Joint Conference of the IEEE Computer and Communications Societies (INFOCOM),Miami, FL, United States, 2005, pp. 878-890.

[8] S. Lindsey , C.S. Raghavendra ,PEGASIS: Power Efficient Gathering in Sensor Information Systems, IEEE Aerospace Conference Proceedings, vol.3 pp.11251130,2002 .

[9] Georgios Samaragdakis, Ibraham Matta and Azer Bestavros, "SEP: A Stable Election Protocol for Clustered heterogeneous wireless sensor networks", pp $1-11,2004$.

[10] Li Qing, Quinqxin Zhu, and Mingwen Wang, "Design of a distributed energy efficient clustering algorithm for heterogeneous wireless sensor networks", Computer communications Journal Elsevier, vol.29, issue12, pp.2230-2237, 2006.

[11] ParulBakaraniya, Sheetal Mehta, "K-LEACH- An Improved LEACH Protocol for Lifetime Improvement in WSN ",IJETT,vol.4,issue5, May 2013.

[12] Sudhanshu Tyagi, Sumit Kumar Gupta, "EHE-Enhanced Hetrogeneous LEACH protocol or lifetime enhancement of wireless sensor networks", IEEE International Conference on Advances in Computing, Communications and informatics, 2013. 